\title{
Multi-wavelength Data Analysis of ONC X-ray Sources
}

\author{
Susan M. Hojnacki*, Nicolas Grosso ${ }^{\dagger}$, Giusi Micela**, Donald Richards ${ }^{\ddagger}$, \\ Norbert Schulz ${ }^{\S}$ and David Huenemoerder ${ }^{\S}$ \\ ${ }^{*}$ California Institute of Technology, 1200 East California Blvd, Pasadena, CA 91125 \\ †Observatoire Astronomique de Strasbourg, 11 rue de l'université, 67000 Strasbourg, France \\ ** INAF, Osservatorio Astronomico G. S. Vaiana, Piazza del Parlamento 1, 90134 Palermo, Italy \\ *Pennsylvania State University, 326 Thomas Building, University Park, PA 16802 \\ ${ }^{\S}$ MIT Kavli Institute for Astrophysics and Space Research, 70 Vassar St, Cambridge, MA 02139
}

\begin{abstract}
To take advantage of the increasing amount of available multi-wavelength astronomical data, we are statistically merging data from several wavelength regimes to analyze astronomical objects. This simultaneous analysis of emission across a wide range of wavelengths will help to provide a composite understanding of young stellar objects. A statistical clustering technique coupled with fused multi-wavelength data from the optical, infrared, and X-ray can provide insight into the physical mechanisms responsible for the intense emission from young stars in different wavelength regimes and can be used to view trends and correlations between those regimes. We present some details of the data fusion followed by our results thus far analyzing spectral data from pre-main sequence (PMS) stars in the Orion Nebula Cluster (ONC).
\end{abstract}

Keywords: <multi-wavelength; young stellar clusters; X-ray emission; statistical methods> PACS: $97.10 . \mathrm{Bt}, 97.21 .+\mathrm{a}, 97.10 .-\mathrm{q}, 95.75 . \mathrm{Pq}$

\section{INTRODUCTION}

The nature and origin of the often variable-intensity emission from young, low-mass stars is an area of intense research in recent years. The debate over the physical mechanisms surrounding emission is having a significant impact on our understanding of the nature of interactions between PMS stars and their planet-forming disks, the timescale over which planets form around Sun-like stars, and the high-energy environment of the early solar system. Despite the vast amount of data now available from previous and present generations of optical, infrared (IR), and X-ray facililities, statistically integrating and combining this data for analysis is not common in the astronomical community and has not yet been undertaken for spectral classification of sources in star formation regions. We have statistically combined data from the optical, IR, and X-ray bands to determine what insights can be gained from using such a multi-wavelength approach. Our research and data analysis of the fused multi-wavelength data has the potential to identify spectral outliers of interest among the sources, classify sources faint in one wavelength band by combination of data from other wavelength regimes, determine if spectral diagnostics can be established that can distinguish between potential emission mechanisms, and search for trends and correlations between wavelength regimes. 


\section{DATA}

To obtain a baseline for the analysis, the initial requirement was that data exist in optical, IR, and X-ray bands for each source. Therefore a complete case analysis was performed with a sample initially limited to T-Tauri stars. The classification algorithm was used solely on the spectral information from observations of the ONC. Data has been collected from the HST Treasury Cycle program for the ONC [2] for optical bands B, V, H-alpha, I, and z-band. J, H, and K band data is from CTIO [4]. X-ray data is from the Chandra Orion Ultradeep Project (COUP, [5]). The result is a conservative, "uncontaminated", robust sample of 213 ONC PMS sources.

\section{PROCEDURE AND ANALYSIS}

Principal Component Analysis (PCA) was used to reduce the dimensionality of the dataset and remove correlation between adjacent bands. PCA can uncover new variables, called principal components (PCs), which account for the majority of the variance in the data. There are no parameters or models to tweak and no coefficients to adjust based on user experience. The answer is based on the data itself and is independent of the user.

PCA was run on the dataset of 5 optical, 3 IR, and 35 X-ray spectral bands for the 213 sources in the sample. The first four PCs explain $87.9 \%$ of the variance and were retained. Two unsupervised clustering methods, agglomerative hierarchical clustering and K-means clustering, were applied sequentially to the 4 PCs to determine a "natural" partitioning of the sample into a number of relatively homogeneous groups or "clusters" of sources [1]. This yielded 18 clusters in the final partition of sources.

The spectral cluster averages were computed for optical and near-IR properties of the population of the ONC for sources where these data exist. The spectral clusters form sequences in $\log$ absorbing column density $\left(\log N_{H}\right)$ and $\log$ effective temperature (log $T_{\text {eff }}$ ). These properties have been determined independently of our research [5].

Infrared excess, an accretion disk indicator, was examined using an index based on IR colors. $\triangle(H-K)$ was used rather than $\triangle(I-K)$ to avoid errors introduced by photometric variability since the optical and IR data were not obtained simultaneously. The spectral clusters form a sequence in $\triangle(H-K)$ (see Fig. 1).

Fig. 2 shows the relationship between $\log N_{H}$ and PC1 for the sources in the sample. There are two prominent outliers in Fig. 2: COUP 948 and COUP 224. COUP 948 is an optical jet source called the Beehive Proplyd. The spectrum in the X-ray has a very unusual, double-peaked spectral energy distribution with prominent soft components indicative of strong shocks in the jet collimation region (Kastner et al. 2005). COUP 224 is a heavily-veiled, hard X-ray source with strong Fe XXV emission at $6.7 \mathrm{keV}$. It is a probable M-type source with equivalent width of the $\lambda 8498 \mathrm{Ca}$ II line of $36 \AA$, indicating possible vigorous accretion [6].

Fig. 3 shows a plot of mean $\log N_{H}$ versus mean EM weighted-average of the thermal plasma temperature for each cluster with these data. A trend can be seen between cluster position on the plot and these parameters. The trends between cluster number and physical properties indicate that the algorithm effectively sorts young stars into physically meaningful clusters. 


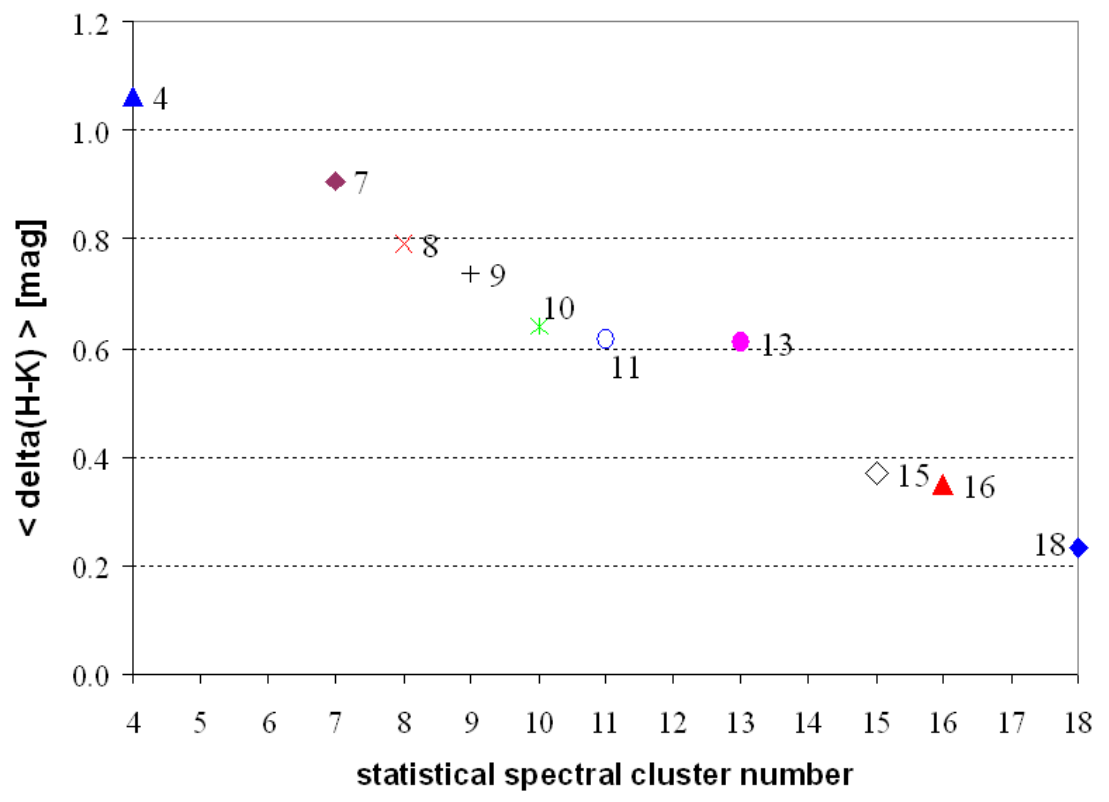

FIGURE 1. Mean K-band excess plotted against cluster number for clusters with sufficient data. Points are labeled with cluster number.

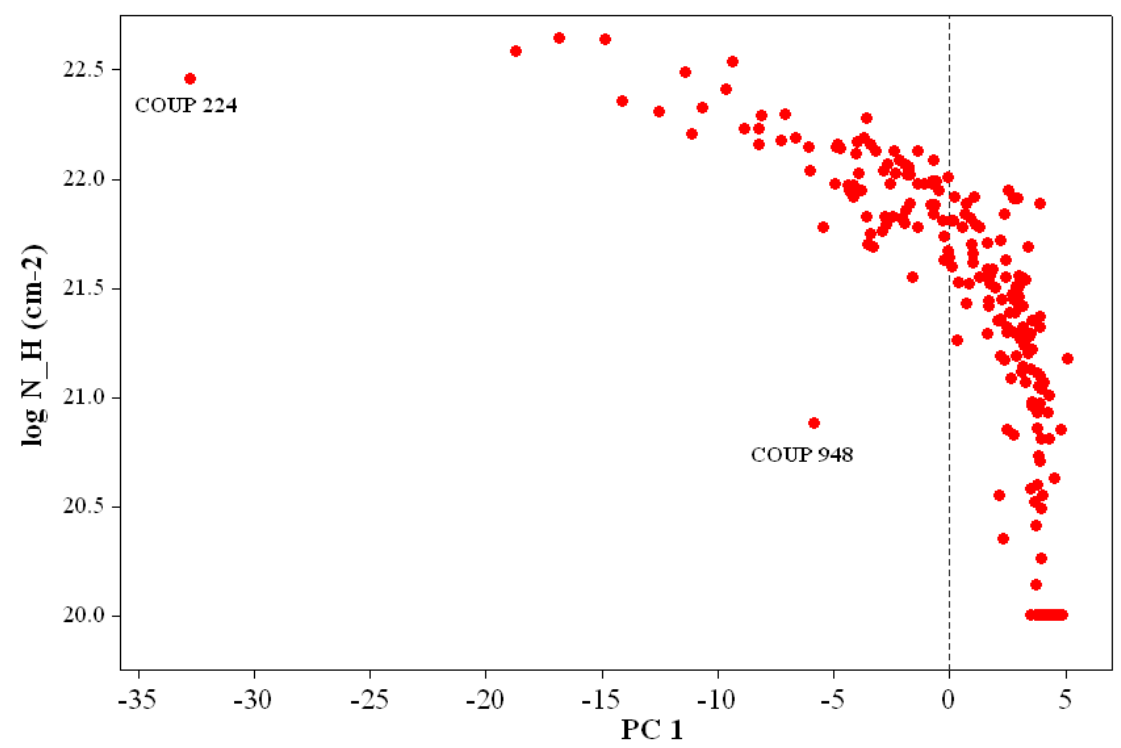

FIGURE 2. Relationship between log absorbing column density and PC1. Outliers are labeled with COUP Id. 


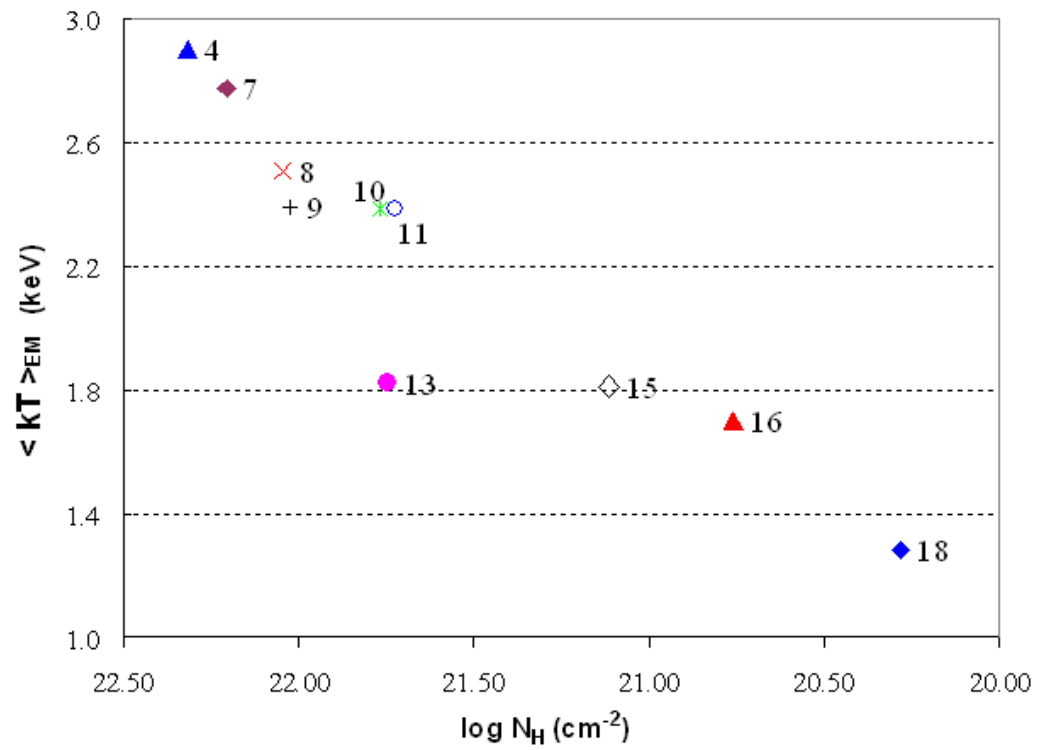

FIGURE 3. Correlation between mean log absorbing column density and mean thermal plasma temperature for each cluster with these data. Points are labeled with cluster number.

\section{ACKNOWLEDGMENTS}

This research is supported by the National Aeronautics and Space Administration under Grant NNX08AM55G issued through the Astrophysics Data Analysis Program to the California Institute of Technology.

\section{REFERENCES}

1. S. M. Hojnacki, J. H. Kastner, G. Micela, E. D. Feigelson, \& S. M. LaLonde, The Astrophysical Journal, 659, 585-598 (2007).

2. M. Robberto, IAU 227 Proceedings, 358-363 (2005).

3. J. H. Kastner, G. Franz, N. Grosso, J. Bally, M. J. McCaughrean, K. Getman, E. D. Feigelson, \& N. S. Schulz, The Astrophysical Journal Supplement, 160, 511-529 (2005).

4. M. Robberto, in prep (2009).

5. K. V. Getman, E. Flaccomio, P. S. Broos, \& 21 other authors, The Astrophysical Journal Supplement, 160, 319-352 (2005).

6. F. C. Riddick, P. F. Roche, \& P. W. Lucas, Monthly Notices of the Royal Astronomical Society, 381, 1077-1092 (2007). 